\title{
Tratamiento de la colecistocoledocolitiasis: colangiografía retrógrada endoscópica preoperatoria versus transoperatoria en el Hospital Miguel Hidalgo de Aguascalientes
}

\author{
Management of cholecystcholedocholithiasis: preoperative versus transoperative \\ endoscopic retrograde cholangiography in Hospital Miguel Hidalgo in Aguascallientes
}

\author{
Claudia Teresa Barba Valadez,* Leonardo Alberto Barba Valadez,** \\ Carlos Martín Gaitán Mercado,* Jorge Luis López Rodríguez***
}

\begin{abstract}
Palabras clave:
Colecistolitiasis, coledocolitiasis, colangiografía retrógrada endoscópica.
\end{abstract}

Key words: Cholecystolithiasis, choledocholithiasis, endoscopic retrograde reatography

Abreviaturas:

$\mathrm{CPRE}=$

Colangiopancreatografía retrógrada endoscópica. ERCP $=$ Endoscopic retrograde

olangiopancreatography.

* Cirujano General. Centenario Hospital Miguel Hidalgo.

Aguascalientes, México.

** Médico residente de segundo año. Hospital de Especialidades del Centro Médico Nacional Siglo XXI, Ciudad de México, México.

*** Cirujano General. Jefe del Departamento de Cirugía. Centro de Ciencias de la Salud. Universidad Autónoma de Aguascalientes.

Aguascalientes, México.

Recibido: 17/07/2017 Aceptado: 27/10/2017

\section{RESUMEN}

Introducción: En la actualidad, existen diferentes estrategias diagnósticas y terapéuticas para abordar la colecistocoledocolitiasis; sin embargo, no existe consenso en cuanto al abordaje terapéutico ideal. El objetivo de este estudio fue comparar los resultados de la exploración endoscópica y la colecistectomía laparoscópica simultánea con la colangiopancreatografía retrógrada endoscópica (CPRE) previa a la colecistectomía por vía laparoscópica. Material y métodos: Estudio ambispectivo, comparativo; se incluyeron los pacientes sometidos a CPRE preoperatoria y aquellos con colecistectomía laparoscópica con CPRE simultánea. Se crearon dos grupos: control (pacientes sometidos a CPRE previa a la colecistectomía laparoscópica) y de estudio (pacientes con realización de CPRE y colecistectomía laparoscópica en un mismo tiempo quirúrgico). Se analizaron variables sociodemográficas y bioquímicas, la estancia hospitalaria, morbilidad y mortalidad. Resultados: Se incluyeron 98 pacientes, 68 en el grupo control y 30 en el grupo de estudio. La tasa de CPRE fallida fue de 5.9\% $(n=4)$ en el grupo control y $3.3 \%(n=1)$ en el grupo de estudio $(\mathrm{p}=0.59)$. Se presentaron complicaciones en el 22.4\% ( $\mathrm{n}=22)$ de la población total, 17 pacientes $(17.3 \%)$ las manifestaron en el grupo control y cinco (5.1\%) en el grupo de estudio $(\mathrm{p}=0.3)$. En el grupo control, la estancia hospitalaria media fue de 7.3 días (3-39 días) y en el grupo de estudio, de 5.3 días (2-11 días) $(p=0.5)$. Conclusión: La colecistectomía laparoscópica simultánea a la exploración de vía biliar con CPRE ofrece una oportunidad de tratamiento definitivo de la colecistocoledocolitiasis, sometiendo al paciente a un solo procedimiento quirúrgico y anestésico.

\section{ABSTRACT}

Introduction: Currently there are different diagnostic and therapeutic strategies to treat cholecystcholedocholithiasis; there is no consensus on the ideal therapeutic approach. The aim of this study was to compare the results of endoscopic examination and simultaneous laparoscopic cholecystectomy with endoscopic retrograde cholangiopancreatography (ERCP) prior to laparoscopic cholecystectomy. Material and methods: Ambispective and comparative study. Patients underwent preoperative ERCP, and patients with simultaneous ERCP and laparoscopic cholecystectomy were included. Two groups were created: control (patients undergoing ERCP prior to laparoscopic cholecystectomy) and study (patients with ERCP and laparoscopic cholecystectomy in the same surgical time). Demographic and biochemical variables, hospital stay, morbidity and mortality were analyzed. Results: We included a total of 98 patients, 68 in the control group and 30 in the study group. The failed ERCP rate was $5.9 \%$ (n $=4)$ in the control group and 3.3\% $(n=1)$ in the study group. ( $p=0.59)$. We registered complications in $22.4 \%$ ( $n$ $=22)$ of the total population: seventeen patients $(17.3 \%)$ in the control group and five (5.1\%) in the study group ( $p$ $=0.3)$. In the control group, mean hospital stay was 7.3 days (3-39 days), and in the study group, 5.3 days (2-11 days) $(p=0.5)$. Conclusion: The simultaneous exploration of the bile duct with ERCP laparoscopic cholecystectomy provides an opportunity for definitive treatment of cholecystcholedocholithiasis. It offers the possibility of definitive treatment in a same surgical and anesthetic time. 


\section{ANTECEDENTES}

E I tratamiento estándar de oro para la colecistolitiasis es la colecistectomía laparoscópica, mientras que el tratamiento para la litiasis aislada de los conductos biliares es el barrido endoscópico. ${ }^{1-3}$ Por el contrario, cuando la litiasis vesicular y la coledocolitiasis están presentes simultáneamente, el abordaje es un reto, ya que no existe consenso en cuanto al manejo ideal. ${ }^{4,5}$ El tratamiento debe ser seguro, eficaz, aplicable y costo-eficiente. ${ }^{6-9}$

A pesar de tener bien determinados los beneficios de la colangiopancreatografía retrógrada endoscópica (CPRE) con colecistectomía laparoscópica, el actual auge por las técnicas avanzadas de laparoendoscopia ha arrojado una diversa cantidad de opciones terapéuticas para la coledocolitiasis: CPRE precolecistectomía, exploración laparoendoscópica y colecistectomía laparoscópica simultánea, exploración abierta o laparoscópica de la vía biliar y CPRE intra- o postcolecistectomía. 7,8

La colangiopancreatografía retrógrada endoscópica está bien establecida como un medio altamente eficiente en despejar la vía biliar en aproximadamente el $90 \%$ de casos. Tiene una tasa de morbilidad del 5 al $9.8 \%$ y una tasa de mortalidad de 0.3 a $2.3 \%$, sobre todo debido a la pancreatitis aguda postoperatoria, perforación y hemorragia duodenal. ${ }^{8}$

En general, la CPRE se ha asociado con dos importantes problemas: falla en la canulación y pancreatitis post-CPRE. El otro inconveniente de la CPRE preoperatoria es que el paciente es sometido a dos procedimientos diferentes, con dos sesiones de anestesia. ${ }^{9}$

Por otro lado, cuando la colecistectomía es realizada mediante una laparotomía, practicar una CPRE preoperatoria incrementa la morbimortalidad, la estancia del paciente y los costos de atención, por lo que actualmente no debe considerarse un procedimiento adecuado para tratar la coledocolitiasis en el preoperatorio si se planea un posterior abordaje quirúrgico convencional. ${ }^{4} \mathrm{Al}$ respecto de lo anterior, se ha descrito la exploración laparoendoscópica, la cual combina durante la colecistectomía laparoscópica la exploración y extracción endoscópica de los litos contenidos en el interior de la vía biliar por CPRE; tiene la ventaja de disminuir tanto la complejidad técnica como el tiempo quirúrgico del procedimiento laparoscópico; de igual forma, se disminuye la morbilidad asociada con la exploración laparoscópica de la vía biliar. ${ }^{9-12}$ Aunque una revisión sistemática de ensayos clínicos aleatorizados ha demostrado que la colecistectomía abierta con exploración de la vía biliar tiene la incidencia más baja de litiasis residual, está asociada con altas tasas de morbilidad y mortalidad, sobre todo en pacientes ancianos. ${ }^{13}$

La técnica de rendez-vous para el acceso a la vía biliar consiste en la introducción de una guía en forma anterógrada (transhepática o transcística) y su posterior captura en el duodeno con el fin de facilitar la canulación de la ampolla de Vater; la maniobra disminuye el tiempo de canulación y las complicaciones asociadas a la manipulación endoscópica de la ampolla de Vater duodenal. ${ }^{10}$

Durante la colecistectomía laparoscópica, la exploración y extracción endoscópica de los litos contenidos en el interior de la vía biliar por CPRE tienen la ventaja de disminuir tanto la complejidad técnica como el tiempo quirúrgico del procedimiento laparoscópico. De igual forma, se reducen las posibilidades de morbilidad asociada con la exploración laparoscópica de la vía biliar. ${ }^{12}$

Recientemente, se ha propuesto llevar a cabo el aclaramiento de la vía biliar por CPRE y esfinterotomía endoscópica durante la colecistectomía laparoscópica para mejorar el cumplimiento del paciente y los resultados clínicos; es más segura y menos costosa. ${ }^{13}$ La principal desventaja de la técnica laparoendoscópica es el paso del endoscopio con el paciente en posición supina. Otros aspectos que han influido en la falta de aceptación de esta técnica incluyen la necesidad de tener al equipo de endoscopia estéril en un entorno de trabajo en equipo, la falta de suministros, equipo de fluoroscopia subóptimo y la necesidad de coordinar los horarios de cirujanos y endoscopistas. $^{10-14}$

Consideramos que la realización de CPRE intraoperatoria resulta ideal, ya que permite el tratamiento en un tiempo bajo un solo procedimiento anestésico. El objetivo del estudio fue comparar los resultados de la exploración endoscópica y la colecistectomía laparoscópica 
simultánea con la CPRE previa a la colecistectomía por vía laparoscópica como tratamiento de la colecistocoledocolitiasis en el Centenario Hospital Miguel Hidalgo.

\section{MATERIAL Y MÉTODOS}

Estudio ambispectivo, comparativo, en el que se incluyeron los pacientes con diagnóstico preoperatorio de colecistitis aguda o crónica, o pancreatitis biliar con coledocolitiasis documentada —el cual estuvo basado en la combinación de los signos clínicos (ictericia, cólico biliar), parámetros bioquímicos de función hepática (elevación de bilirrubinas total a expensas de bilirrubina directa, fosfatasa alcalina, gamma glutamil transpeptidasa) y ultrasonido abdominal con evidencia de litos en colédoco o dilatación de vía biliar-sometidos a CPRE preoperatoria, así como aquellos con colecistectomía laparoscópica con exploración endoscópica de vía biliar, durante el periodo comprendido del 01 de enero de 2009 al 01 de noviembre de 2014, en el Centenario Hospital Miguel Hidalgo. Los pacientes del estudio fueron clasificados en dos grupos: control (conformado por los pacientes sometidos a CPRE previa a la colecistectomía laparoscópica) y de estudio (formado por los pacientes con realización de CPRE y colecistectomía laparoscópica en un mismo tiempo quirúrgico).

Se realizó un análisis de las variables sociodemográficas como el sexo y la edad, además de los niveles séricos de bilirrubinas totales, bilirrubina directa, fosfatasa alcalina y gamma glutamil transpeptidasa. Las variables de interés a comparar entre ambos grupos fueron los días de estancia hospitalaria, complicaciones mayores (conversión a cirugía abierta, pancreatitis post-CPRE, lesión de vía biliar, fuga biliar, hemorragia, lesión intestinal o de víscera hueca, litiasis residual, infección de sitio quirúrgico), complicaciones menores (hernia incisional, reingreso hospitalario), mortalidad, intervalo entre el ingreso hospitalario y el tratamiento quirúrgico definitivo, intervalo entre el ingreso y la realización de la CPRE, días transcurridos entre la realización de la CPRE y el tratamiento quirúrgico.

Para el análisis de la información, se realizó una base de datos en el programa SPSS versión
21.0. Se aplicó estadística descriptiva para variables cuantitativas y empleo de medidas de tendencia central y dispersión de acuerdo a la distribución de los datos para las variables cualitativas, frecuencias y porcentajes. Fue aplicada la estadística inferencial para la comparación de grupos y variables dicotómicas; $\chi^{2}$ para variables cualitativas, t de Student para las cuantitativas y $U$ de Mann-Whitney para variables cuantitativas de distribución libre; se consideró un valor de $p<0.05$ con significancia estadística.

\section{RESULTADOS}

Un total de 98 pacientes fueron incluidos en el estudio y clasificados en dos grupos: 68 en los que se utilizó CPRE preoperatoria más colecistectomía laparoscópica (grupo control) y 30 en los que se intervino mediante CPRE intraoperatoria y colecistectomía laparoscópica (grupo estudio).

El promedio de edad de la población fue de $37.9 \pm 17.3$ años (rango: 16-82 años). La distribución por sexo tuvo una relación de 4.7:1 femenino-masculino, siendo $17.3 \%(n=17)$ del sexo masculino y $82.7 \%(n=81)$ del sexo femenino (Cuadro I).

La media del diámetro del conducto colédoco en la población total fue de $9.2 \pm$ 2.8 milímetros (rango: 3-15 mm). En el grupo control, de 9.2 milímetros (rango: 3-15) y en el grupo de estudio, de $9.3 \mathrm{~mm}$ (rango 4-15 mm).

El $89.7 \%(n=88)$ de los pacientes presentaron elevación de bilirrubina total, con rangos de 0.18 a $13.9 \mathrm{mg} / \mathrm{dl}$ y una mediana de 4.3. El 96.9\% ( $n=95)$ manifestó elevación de bilirrubina directa en un rango de $0.04 \mathrm{a}$ $11 \mathrm{mg} / \mathrm{dl}$, con una mediana de $2.8 \mathrm{mg} / \mathrm{dl}$. Ochenta y seis pacientes $(87.7 \%)$ presentaron elevación de fosfatasa alcalina con un rango de 84 a 1,124 U/l, y una mediana de 235 $\mathrm{U} / \mathrm{l}$. Noventa y tres $(94.8 \%)$ de los pacientes tuvieron elevación de gamma glutamil transpeptidasa con rangos de cero a 1,400 UI/l, mediana de $330 \mathrm{U} / \mathrm{l}$. No hubo significancia estadística para ninguna de las variables de los parámetros enzimáticos (Cuadro II).

De los 98 pacientes incluidos, en 78 (79.6\%) el diagnóstico postquirúrgico fue coledocolitiasis y en los restantes 20 (20.4\%) no se 
Cuadro I. Distribución por edad y sexo de los pacientes por grupo.

\begin{tabular}{lcccc} 
& Control & Estudio & Total & $\mathbf{p}$ \\
\hline $\begin{array}{l}\text { Edad (años) } \\
\text { Promedio }\end{array}$ & 37.1 & 39.9 & & \\
Rango & $16-82$ & $18-75$ & $16-8$ & 0.91 \\
Sexo & & & & \\
Femenino & $55(67.9 \%)$ & $26(32.1 \%)$ & $81(82.7 \%)$ & 0.48 \\
Masculino & $4(23.5 \%)$ & $13(76.5 \%)$ & $17(17.3 \%)$ &
\end{tabular}

Cuadro II. Distribución de los parámetros bioquímicos y diámetro del colédoco por grupo.

\begin{tabular}{lcccc} 
& $\begin{array}{c}\text { Control } \\
\boldsymbol{X} / \mathbf{M}\end{array}$ & $\begin{array}{c}\text { Estudio } \\
\boldsymbol{X} / \mathbf{M}\end{array}$ & $\begin{array}{c}\text { Total } \\
\boldsymbol{X} / \mathbf{M}\end{array}$ & $\mathbf{p}$ \\
\hline Diámetro del colédoco (mm) & 9.2 & 9.3 & $9.2 \pm 2.8$ & 0.83 \\
Bilirrubina total (mg/dl) & $4.3(0.45-13.9)$ & $4.3(0.18-11.9)$ & $4.3(0.18-13)$ & 0.90 \\
Bilirrubina directa (mg/dl) & $2.6(0.04-11)$ & $3.2(0.10-9.74)$ & $2.8(0.04-11)$ & 0.45 \\
Fosfatasa Alcalina (U/l) & $247.5(84-1,124)$ & $215.5(84-927)$ & $235(84-1,124)$ & 0.96 \\
GGT (U/l) & $330.0(0-1,377)$ & $320.6(0-1,400)$ & $330(0-1,400)$ & 0.77
\end{tabular}

logró la evidencia de litos en el conducto biliar $(p=0.2)$. Independientemente del diagnóstico postquirúrgico, todos los pacientes fueron sometidos a CPRE. La eficacia de la CPRE obtuvo un valor de $94.1 \%(n=93)$, con un $5.1 \%$ de falla $(n=5)$, siendo imposible la canulación en el primer intento. De ellos, cuatro pacientes $(5.9 \%)$ pertenecían al grupo control y uno $(3.3 \%)$ al grupo de estudio $(p=0.59)$. La conversión a cirugía abierta fue necesaria en cuatro pacientes (4.1\%).

Se presentaron en el grupo control cinco complicaciones de 30 pacientes, lo que dio un $16.7 \%$; en el grupo de estudio fueron 17 complicaciones de un total de 68 pacientes, lo que representó un 25\% (Figura 1).

Dentro de las complicaciones encontradas, la pancreatitis post-CPRE se presentó en 14 pacientes (14.2\%) (Cuadro III). No hubo reingresos hospitalarios ni defunciones.

En la variable de estancia hospitalaria, el grupo control tuvo una media de 7.3 días (3-39 días), y el grupo de estudio, una media de 5.3 días (2-11 días); $p=0.5$. En los días transcurridos del ingreso a la realización de la
CPRE, el grupo control tuvo una media de 2.8 días y el grupo de estudio de tres días, con una $p=0.3$. En relación con los días transcurridos del ingreso al tratamiento definitivo, el grupo control presentó una media de 6.7 días y el grupo de estudio una de tres días, con una $\mathrm{p}$ $=0.03$, con significancia estadística. Con respecto a los días transcurridos de la realización de la CPRE al manejo quirúrgico definitivo, en el grupo de estudio se realizó en el mismo tiempo quirúrgico y para el grupo control hubo una media de 3.9 días, con lo que se obtuvo una $\mathrm{p}=0.002$ (Cuadro IV).

\section{DISCUSIÓN}

Diversos estudios ${ }^{3,4}$ muestran que existe una mayor incidencia de colelitiasis en el sexo femenino que en el masculino; de igual forma, la prevalencia aumenta a partir de los 40 años. Estos datos son similares en los encontrados en nuestra población, donde el promedio de edad fue de $37.9 \pm 17.3$ años y la relación de frecuencia y sexo fue de 4.7:1 femeninomasculino. 
Hace más de 10 años, Cuschieri y sus colaboradores, ${ }^{15}$ en un ensayo multicéntrico aleatorizado, demostraron que la remoción endoscópica preoperatoria con CPRE seguida de colecistectomía laparoscópica fue comparable a la colecistectomía laparoscópica con

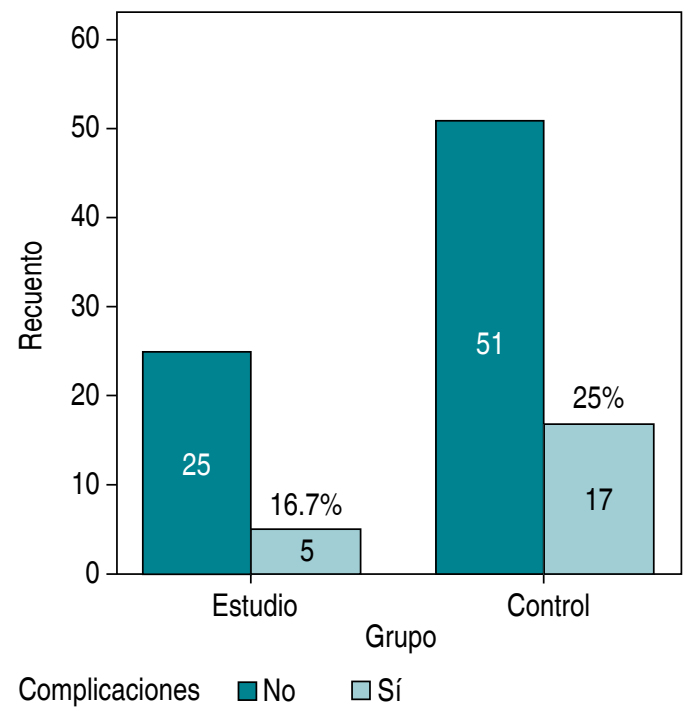

Figura 1. Distribución de la presentación de complicaciones por grupo.
CPRE intraoperatoria en términos de tasa de éxito, morbilidad y mortalidad. Esto, además, se ha demostrado en estudios posteriores, ${ }^{16}$ que también establecen que no hay reducción significativa en el número de litos retenidos y las tasas de fracaso en los grupos de CPRE pre- e intraoperatoria.

Aunque el perfil bioquímico anormal se ha utilizado como tamizaje de pacientes que requieren CPRE transoperatoria, los valores anormales de las pruebas bioquímicas en suero no son ni sensibles ni específicos. Incluso en pacientes en los que se habían elevado valores en todas las pruebas bioquímicas, solamente en el $30 \%$ de ellos fue posible comprobar la presencia de coledocolitiasis mediante CPRE. ${ }^{17}$ En este trabajo fue posible medir diversos valores séricos sin encontrar significancia estadística alguna entre los grupos; por otro lado, no se buscó una asociación entre la confirmación de los valores bioquímicos y la presencia o no de la coledocolitiasis, aunque se ha sugerido que los niveles de GGT superiores a siete veces los valores normales pueden predecir la presencia de litiasis en el árbol biliar. ${ }^{18}$

Otras investigaciones ${ }^{19}$ reportan que solo en $27 \pm 54 \%$ de los pacientes con sospecha de

\section{Cuadro III. Distribución de complicaciones por grupo.}

\begin{tabular}{lcll}
\multicolumn{1}{c}{ Complicaciones } & Control & Estudio & p \\
\hline Pancreatitis post-CPRE & $11(11.02 \%)$ & $3(3.05 \%)$ & 0.4 \\
Litiasis residual & $5(5.1 \%)$ & $2(2 \%)$ & 0.9 \\
Lesión intestinal & $1(1.02 \%)$ & 0 & 0.1 \\
Sepsis & $1(1.02 \%)$ & 0 & 0.1 \\
Reintervención & $1(1.02 \%)$ & 0 & 0.1
\end{tabular}

Cuadro IV. Distribución de días de estancia

hospitalaria y días transcurridos al manejo definitivo por grupo.

\begin{tabular}{lccl} 
& Grupo control & Grupo estudio & p \\
\hline Días de estancia hospitalaria total & 7.3 & 5.3 & 0.5 \\
Días del ingreso a la realización de CPRE & 2.8 & 3 & 0.3 \\
Días del ingreso al tratamiento definitivo & 6.7 & 3 & 0.03 \\
Días de la CPRE al tratamiento quirúrgico & 3.9 & 0 & 0.002
\end{tabular}


litos en el conducto biliar común basada en las pruebas bioquímicas se encuentran realmente coledocolitiasis durante la CPRE, mientras que en nuestro estudio obtuvimos un $79.6 \%$ de diagnóstico de coledocolitiasis durante la realización de la CPRE con sospecha previa de litiasis; sin embargo, es necesario considerar la distinción entre litos, lodo biliar y hasta estenosis, edema o, incluso, una ampolla de váter desflorada, que sugiere indirectamente que pasó un lito que ya no es demostrable, pero que apoya el diagnóstico de coledocolitiasis. Es necesario aclarar que la CPRE preoperatoria es la menos costo-eficiente debido al elevado índice de colangiografías endoscópicas normales con ausencia de coledocolitiasis, hecho que se asocia a las limitaciones propias de los factores predictivos de esta. ${ }^{6}$

En nuestro estudio, el diámetro promedio del conducto colédoco fue de $9.2 \pm 2.8$ milímetros (rango: 3-15 mm); es decir, la mayoría de los pacientes tenían dilatación de la vía biliar que apoyaba el diagnóstico de coledocolitiasis. Los casos que no fueron así, tenían sintomatología y niveles bioquímicos que justificaban la realización de una CPRE.

Obtuvimos un $5.1 \%$ de falla en la canulación durante la realización de la CPRE, lo cual es similar a lo descrito por Testoni en una revisión sobre la dificultad en la canulación de la vía biliar. ${ }^{20}$

La morbilidad (22.4\%) detectada en nuestro trabajo fue elevada en comparación con otras investigaciones, donde se describe del $6.6 \%$ al $15.4 \% .{ }^{21,22}$ Aunque en diversos estudios también se presenta una mayor tasa de complicaciones en los pacientes que se someten a CPRE preoperatoria, no se ha logrado establecer una diferencia significativa entre la morbilidad observada en el procedimiento de dos etapas (CPRE preoperatoria + colecistectomía) y el de una sola etapa. ${ }^{22}$

La pancreatitis es la complicación más frecuente de la colangiopantografía retrógrada endoscópica, con rangos reportados de 1.8 a $7.2 \%$. Su frecuencia varía ampliamente y depende de diferentes factores, tales como la técnica utilizada para la canulación y el número de pacientes tratados anualmente. ${ }^{23,24}$ En nuestro trabajo, se presentó en $14.2 \%$ de nuestros pacientes: el doble de lo reportado en otros trabajos; no obtuvimos diferencias significativas entre los grupos, pero se observó una mayor tendencia a la presentación de pancreatitis en el grupo de CPRE preoperatoria. Asimismo, tampoco se encontraron diferencias entre los dos grupos con respecto a la litiasis residual, y obtuvimos un porcentaje similar al descrito en otros estudios, en los cuales reportan de 2 a $15 \% .^{25}$

No existieron casos de lesión de vía biliar o defunciones, lo cual concuerda con investigaciones como la de Swahn, ${ }^{26}$ donde describe 122 pacientes con canulación exitosa de la vía biliar y bajo porcentaje de litiasis residual en un estudio comparativo de CPRE previa y durante la colecistectomía.

Una de las ventajas del uso de la CPRE intraoperatoria puede incluir la identificación de los cálculos del conducto biliar común no detectados previamente, así como una mejor definición de la anatomía ductal extrahepática, lo que ayudará al cirujano para evitar daños accidentales a la vía biliar. ${ }^{27}$ Por otra parte, puede ocurrir la lesión de la vía extrahepática, incluso en la mano experta de los cirujanos laparoscópicos que abogan por el uso rutinario de la CPRE transoperatoria; por lo tanto, el empleo de rutina de esta técnica no proporciona seguridad para evitar la lesión de la vía extrahepática. ${ }^{21,22,26}$

$\mathrm{Al}$ analizar la variable de días transcurridos del ingreso al tratamiento definitivo encontramos una diferencia significativa entre los grupos de estudio; es obvio que al realizar la colecistectomía en el mismo acto quirúrgico y anestésico que la realización de la CPRE, disminuirá este lapso; sin embargo, en relación con la estancia hospitalaria global, no hallamos una significancia estadística, lo que indica que los pacientes se mantenían hospitalizados a pesar de la resolución de su padecimiento. Esto podría estar dado por la saturación en el uso del endoscopio para la realización de la CPRE y no en el tiempo quirúrgico para la colecistectomía.

Diversos reportes ${ }^{13-16}$ muestran que la estadía en el hospital y el costo total del tratamiento son mucho menores con el grupo de laparoscopia de una etapa, lo que ha llevado a diversos autores a recomendar este abordaje como el procedimiento de 
elección. Una de las limitaciones importantes para la realización de este trabajo fue una falta de coordinación entre los equipos de endoscopia y quirúrgico para llevar a cabo el tratamiento con CPRE y colecistectomía en un mismo tiempo; por lo anterior, la diferencia intrahospitalaria entre ambos grupos no resultó significativa.

\section{CONCLUSIÓN}

El mejor momento para la exploración de la vía biliar es hacerla en conjunto con el procedimiento quirúrgico de la colecistectomía laparoscópica, ya que ofrece la oportunidad de tratamiento definitivo en un mismo tiempo quirúrgico y anestésico.

\section{REFERENCIAS}

1. Sekimoto $M$, Takada $T$, Kawarada $Y$, Nimura $Y$, Yoshida M, Mayumi T, et al. Need for criteria for the diagnosis and severity assessment of acute cholangitis and cholecystitis: Tokyo Guidelines. J Hepatobiliary Pancreat Surg. 2007; 14: 11-14.

2. Barreto SE, Soler PL, Sugrañes MA. Coledocolitiasis diagnóstico y terapéutica mediante la colangiopancreatografía retrógrada endoscópica. AMC. 2010; 14: 1-11.

3. Almadi MA, Barkun JS, Barkin AN. Management of suspected stones in the common bile duct. CMAJ. 2012; 184: 884-892.

4. Hoyuela C, Cugat E, Marco C. Opciones actuales para el diagnóstico y tratamiento de la coledocolitiasis. Cir Esp. 2000; 68: 243-253.

5. Nakeeb A, Comuzzie A, Martin L, Sonnenberg GE, Swartz-Basile D, Kissebah $\mathrm{AH}$, et al. Gallstones: genetics versus environment. Ann Surg. 2002; 235: 842-849.

6. Pekolj J. Tratamiento de la litiasis coledociana por vía laparoscópica. Continúa la controversia. Cir Esp. 2012; 90: 144-146.

7. Poulose BK, Speroff T, Holzman MD. Optimizing choledocholithiasis management: a cost-effectiveness analysis. Arch Surg. 2007; 142: 43-48.

8. Sharma A, Dahiya P, Khullar R, Soni V, Baijal M, Chowbey PK. Management of common bile duct stones in the laparoscopic era. Review article. Indian J Surg. 2012; 74: 264-269.

9. Rosen M, Brody F, Ponsky J. Predictive factors for conversion of laparoscopic cholecystectomy. Am J Surg. 2002; 184: 254-258.

10. Tommasi C, Bencini L, Bernini M, Naspetti R, Cavallina G, Manetti R. Routine use of simultaneous laparoendoscopic approach in patients with confirmed gallbladder and bile duct stones: fit for laparoscopy fit for "rendezvous". World J Surg. 2013; 37: 999-1005.

11. Tzovaras G, Baloyiannis I, Zachari E, Symeonidis D, Zacharoulis D, Kapsoritakis A, et al. Laparoendoscopic rendezvous versus preoperative ERCP and laparoscopic cholecystectomy for the management of cholecystocholedocholithiasis. Ann Surg. 2012; 255: 435-439.

12. Vázquez JA, Tarango E, Vázquez AL, Vázquez AR, García O, Guerrero GA. Caso clínico. "Rendezvous" laparoendoscópico para el tratamiento de la colecistocoledocolitiasis. Cir Gen. 2010; 32: 267-269.

13. Perreira F, Graterol Y, Venales-Barrios J, BousquetSuaréz A, Cáceres-Cauro C, Romero-Bravo J, et al. Maniobra de "rendezvous" como una opción técnica de acceso a la vía biliar: Reporte de casos. Rev de Gastroenterol Mex. 2012; 77: 224-228.

14. Gurusamy K, Sahay SJ, Burroughs AK, Davidson BR. Systematic review and meta-analysis of intraoperative versus preoperative endoscopic sphincterotomy in patients with gallbladder and suspected common bile duct stones. Br J Surg. 2011; 98: 908-916.

15. Cuschieri A, Lezoche E, Morino M, Croce E, Lacy A, Toouli J, et al. EAES multicenter prospective randomized trial comparing two-stage vs. single stage management of patients with gallstone disease and ductal calculi. Surg Endosc. 1999; 13: 952-957.

16. Dasari BV, Tan CJ, Gurusamy KS, Martin DJ, Kirk G, McKie L, et al. Surgical versus endoscopic treatment of bile duct stones. Cochrane Database Syst Rev. 2013; 3: CD003327.

17. Silverstein JC, Wavak E, Millikan KW. A prospective experience with selective cholangiography. Am Surg. 1998; 64: 654-659.

18. Prat F, Meduri B, Ducot B, Chiche R, SalimbeniBartolini R, Pelletier G. Prediction of common bile duct stones by noninvasive tests. Ann Surg. 1999; 229: 362-368.

19. Tham TC, Lichtenstein DR, Vandervoort J, Wong RC, Brooks D, Van Dam J, et al. Role of endoscopic retrograde cholangiopancreatography for suspected choledocholithiasis in patients undergoing laparoscopic cholecystectomy. Gastrointest Endosc. 1998; 47: 50-56.

20. Testoni PA, Testoni S, Giussani A. Difficult biliary cannulation during ERCP: how to facilitate biliary access and minimize the risk of post-ERCP pancreatitis. Dig Liver Dis. 2011; 43: 596-603.

21. Rábago LR, Vicente C, Soler F, Delgado M, Moral I, Guerra I, et al. Two-stage treatment with preoperative endoscopic retrograde cholangiopancreatography (ERCP) compared with single-stage treatment with intraoperative ERCP for patients with symptomatic cholelithiasis with possible choledocholithiasis. Endoscopy. 2006; 38: 779-786.

22. Lu J, Cheng Y, Xiong X, Wy SJ, Cheng NS. Two-stage vs. single-stage management for concomitant gallstones and common bile duct stones. World J Gastroenterol. 2012; 18: 3156-3166.

23. Vandervoort J, Soetikno RM, Tham TC, Wong RC, Ferrari AP Jr, Montes H, et al. Risk factors for complications after performance of ERCP. Gastrointest Endosc. 2002; 56: 652-656.

24. Menéndez A, Kleinfinger S, Vidal P, Ramírez C, Kunz W, González M. Riesgo-beneficio de la colangiografía transoperatoria de rutina. Rev Mex Cir Endosc. 2004; 5: 99-103.

25. Lilly MC, Arregui ME. A balanced approach to choledocholithiasis. Surg Endosc. 2001; 15: 467-472. 
26. Swahn F, Regnér S, Enochsson L, Lundell L, Permert J, Nilsson $M$, et al. Endoscopic retrograde cholangiopancreatography with rendezvous cannulation reduces pancreatic injury. World J Gastroenterol. 2013; 19: 6026-6034.

27. Cervantes J, Rojas G. Análisis de la práctica de la colangiografía transoperatoria en un periodo de veinte años. Cir Gen. 2003; 25: 34-40.
Correspondencia:

Dra. Claudia Teresa Barba Valadez

República de Costa Rica Núm. 309-A,

Fraccionamiento Las Américas, 20230,

Aguascalientes, Aguascalientes, México.

Tel.: (449) 1466860

Cel.: (449) 8942592

E-mail: claus.bar@hotmail.com 